\title{
Simulation of Multiphase Flow of the Oil-Water Separation in a Rotating Packed Bed for Oil Purification
}

\author{
Xiaojun Zhang, Yun Cheng, Songlin Nie, Hui Ji, and Laiguo Liu \\ College of Mechanical Engineering and Applied Electronics Technology, Beijing University of Technology, Beijing 100124, China \\ Correspondence should be addressed to Songlin Nie; niesonglin@tom.com
}

Received 12 December 2012; Accepted 22 January 2013

Academic Editor: Xiaosheng Qin

Copyright ( 2013 Xiaojun Zhang et al. This is an open access article distributed under the Creative Commons Attribution License, which permits unrestricted use, distribution, and reproduction in any medium, provided the original work is properly cited.

\begin{abstract}
HIGEE (High Gravity Rotary Device) rotating oil purifier which consists of two parts: hydrocyclone separator and rotating packed bed (abbr. RPB) is considered to be capable of removing the solid particle contaminant, moisture and gas simultaneously. As the major unit of HIGEE, the RPB uses centrifugal force to intensify mass transfer. Because of the special structure of RPB, the hydraulic characteristics of the RPB are very important. In this study, the multiphase flow model in porous media of the RPB is presented, and the dynamical oil-water separation in the RPB is simulated using a commercial computational fluid dynamics code. The operating conditions and configuration on the hydraulic performance of the RPB are investigated. The results have indicated that the separation efficiency of HIGEE rotating oil purifier is predominantly affected by operating conditions and the configurations. The best inlet pressure is $0.002 \mathrm{MPa}$. When the liquid inlet is placed in the outside of the lower surface of RPB; oil outlet is placed in the upper surface, where it is near the rotation axis; and water outlet is placed in the middle of the RPB, where it is far away from the oil outlet, the separating efficiency is the best.
\end{abstract}

\section{Introduction}

The pollution of hydraulic engineering equipment is the mainly responsible for the trouble of hydraulic system, and it is the key of maintenance. Contaminant particles can bring about a number of detrimental effects on the hydraulic system components as well as the fluid itself. One of the key problems of hydraulic contamination control is to design and maintain system reasonably reducing the contamination level of key point as low as possible. There are various kinds of purification technologies to improve the contamination control level of fluid power system (FPS), such as oil filter, electrostatic oil cleaner, oil vacuum cleaning, magnetic field pulse filtration, and other coalescence methods [1]. However, most of traditional purification technologies can only remove individual contamination while there are solid particle contaminant, moisture, and gas simultaneously in the FPS. A novel hydraulic oil purifier is developed to be capable of removing the solid particle contaminant, moisture, and gas simultaneously, which consists of two parts: hydrocyclone separator and rotating packed bed (RPB) as shown in Figure 1.
Rotating packed beds (RPBs) intensify mass transfer by using centrifugal force to realize separation, which has been applied to distillation, absorption, striping, polymer devolatilization, bio-oxidation, and so on [2-6]. HIGEE (high gravity rotary device) rotating oil purifier is based on the traditional oil purification, which is developed on the introduction of the HIGEE technology. The fluid from preliminary purification goes into the internal space of rotating bed along the axis of rotation and rotates at high speed with the rotating packed bed driven by the motor. The function of packing is increasing the speed of fluid, and huge shear force overcomes surface forces, increasing contact area between different phases, tearing fluid into spray of micron order. In the action of HIGEE, when the droplet contains spray through the voids of the layers of packing which rotates at high speed, which is bend, narrow, and varied, which is also filled with very thin spray and very small droplet, the inertia settlement ability of spray and droplet will be enhanced. It will form rapid collision for effective coagulation between droplet and spray, in the action of different physical properties of packing which are hydrophilic or hydrophobic to droplet and spray; finally the media of different density 




FIGURE 1: 3D plot of HIGEE rotating oil purifier.

are separated out in the action of centrifugal force [7].

The hydraulic characteristics of the RPB have been reported in many studies, such as the liquid holdup, the pressure drop, flooding, residence time distribution, and visualized liquid flow [8-11]. Ramshaw and Mallinson conducted a water-oxygen absorption system in an RPB and found that the mass transfer coefficient was 27-44 times higher than that in conventional packed columns [12]. Tung and Mah theoretically proposed a correlation for the mass transfer coefficient in an RPB [13]. Munjal et al. proposed a correlation in an $\mathrm{RPB}$ theoretically and experimentally studied for the absorption of $\mathrm{CO}_{2}$ from air into $\mathrm{NaOH}[14,15]$. Kumar and Gardner obtained mass transfer coefficients in an RPB packed with aluminum foam metal of various specific surface areas in a $\mathrm{CO}_{2}$-water system [16]. In 1990, Kumar and Rao performed experiments of absorption of $\mathrm{CO}_{2}$ from air into $\mathrm{NaOH}$ solution in an RPB and found that the mass transfer coefficient changed with increasing liquid rates and rotation speeds [17]. Singh et al. investigated the mass transfer in an RPB for air stripping of volatile organic compounds from groundwater [18]. Chen et al. also did a lot of research on the RPB, they evaluated the mass transfer coefficient of an oxygen-water absorption system [19], and they investigated the influence of liquid viscosity on the mass transfer rate for both Newtonian and non-Newtonian fluids in an RPB [20]. Further, they evaluated the end effects of an RPB by varying the radii of the packed bed [21]. Burns and Ramshaw took the visual study of liquid flow in RPBs under different rotating speeds. They observed the spiral of liquid and severe liquid maldistribution on radial orientation; in contrast the liquid wandered slightly laterally and consequently led to a relatively uniform distribution on the tangential orientation



Rotating packed bed Hydrocyclone separator

FIGURE 2: Sectional drawing of HIGEE rotating oil purifier. 1: connecting motor shaft; 2 : the hydraulic oil outlet; 3: packing; 4 : vortex finder; 5: underflow outlet; 6: shell; 7: tangential nozzle; 8: contaminated oil inlet; 9: parting face.

[22]. Similar results were reached by the researchers [7] in Beijing University of Chemical Technology [11].

Many studies have been investigated by theory or experiment. However, many physical experiments are very expensive and time-consuming, and there are no precise experimental data about the flow in RPBs. Hence, using mathematical models as design tools can contribute to a better understand of the hydraulic characteristics of the $\mathrm{RPB}$ with the fast development of the computer technology. Computational fluid dynamics (CFD) is a good design and analysis tool to simulate the flow of mass and momentum throughout a fluid continuum. It is an advantage method to study the hydraulics characteristics of RPB. Numerical simulation by using FLUENT software will be conducted in this research. The multiphase flow in porous media of RPB will be numerically studied. The effects of operating conditions and configuration on the hydraulic performance of RPB are investigated to increase the separation efficiency of HIGEE rotating oil purifier.

\section{Mathematical Methods}

2.1. Physical Model. According to the introduction of HIGEE rotating oil purifier (Figure 1), Figure 2 shows the geometrical diagram of HIGEE rotating oil purifier designed for this research, which consists of two parts: hydrocyclone separator and rotating packed bed (RPB). The hydraulic characteristics of RPB are very important. In this study, the multiphase flow model in porous media of RPB is presented.

The 3D model (Figure 3) is developed for the RPB considered using a commercial code Gambit. The diameter of the RPB is $110 \mathrm{~mm}$, and its height is $60 \mathrm{~mm}$. The whole rotating packed bed is taken as the object of study in this research. In Figure 3, the whole rotating packed bed is a cylinder. There are three small cylinders at the upper and lower surfaces of the RPB. The oil and water outlets are at the upper surface and the oil-water mixture inlet is at the lower surface. 


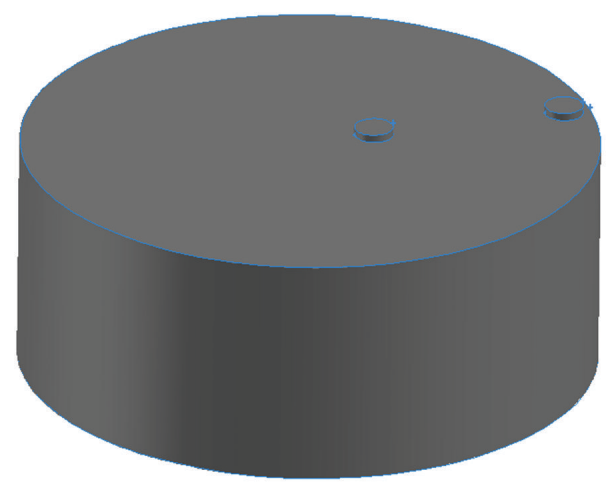

Figure 3: 3D model of the RPB.

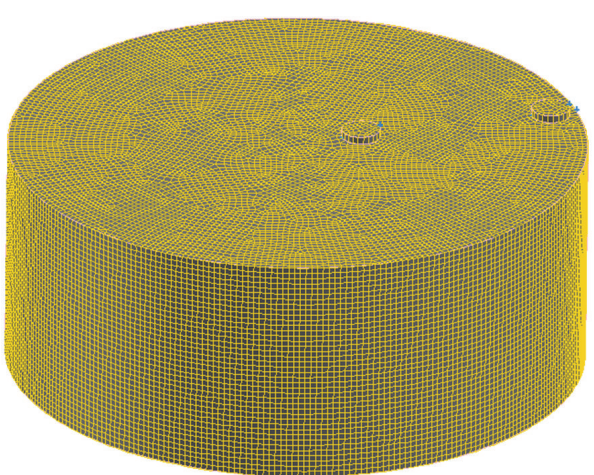

FIGURE 4: Mesh generation of the 3D model.
2.2. Mathematical Model. In this research, the Eulerian multiphase model is applied. The phases are water and oil. The description of multiphase flow as interpenetrating continua incorporates the concept of phasic volume fractions which is defined by $\alpha_{q}$ in this paper. Because there is no temperature gradient, so only the mass conservation and the momentum equations are used. Volume fractions represent the space occupied by each phase, and the laws of conservation of mass and momentum are satisfied by each phase individually [23].

The volume of phase $q$ is $V_{q}$, which is defined by

$$
V_{q}=\int_{V} \alpha_{q} d V
$$

where

$$
\sum_{q=1}^{n} \alpha_{q}=1
$$

The effective density of phase $q$ is

$$
\widehat{\rho_{q}}=\alpha_{q} \rho_{q}
$$

where $\rho_{q}$ is the physical density of phase $q$. Continuity equation is shown as

$$
\nabla \cdot\left(\widehat{\rho_{q}} v\right)=0
$$

where $v$ is the vector velocity of the liquid. Momentum balance equation is shown as

$$
\nabla \cdot\left(\widehat{\rho_{q}} v v\right)=-\nabla \times P+\nabla \cdot\left(\mu\left(\nabla \times v+(\nabla \times v)^{T}\right)\right)
$$

where $P$ is the static pressure, and $\mu$ is the viscosity. Porous media are simulated by adding a momentum source term $S_{i}$ to the standard fluid flow equations; therefore the momentum balance in the porous media could be defined as

$$
\begin{array}{r}
\nabla \cdot\left(\widehat{\rho_{q}} v v\right)=-\nabla \times P+\nabla \cdot\left(\mu\left(\nabla \times v+(\nabla \times v)^{T}\right)\right)+S_{i} \\
(i=x, y, z)
\end{array}
$$

where $S_{i}$ is the source term for the momentum equation, which is composed of two parts: a viscous loss term (the first term on the right-hand side of (7)), and inertial loss term (the second term on the right-hand side of (7)):

$$
S_{i}=-\left(\sum_{j=1}^{3} D_{i j} \mu v_{i}+\sum_{j=1}^{3} C_{i j} \frac{1}{2} \widehat{\rho_{q}}\left|v_{i}\right| v_{i}\right) \quad(i=x, y, z),
$$

where $D$ and $C$ are viscous resistance and inertia loss coefficient matrices respectively. When the case is simple homogeneous porous media, the source term is shown as

$$
S_{i}=-\left(\frac{\mu}{\alpha} v_{i}+C_{2} \frac{1}{2} \hat{\rho}_{q}\left|v_{i}\right| v_{i}\right) \quad(i=x, y, z),
$$

where $\alpha$ is the permeability and $C_{2}$ is the inertial resistance coefficient. $D$ and $C$ are simply specified as diagonal matrices with $1 / \alpha$ and $C_{2}$, respectively $[24,25]$.

In this paper, the Ergun equation is used to derive porous media input for a packed bed, and the laminar flow through the porous media is simulated, which is similar to a packed bed in this paper and easy to simulate on existing computers. The permeability and inertial loss coefficients in each component direction could be identified as

$$
\begin{aligned}
& \alpha=\frac{d^{2}}{150} \frac{\varepsilon^{3}}{(1-\varepsilon)^{2}}, \\
& C_{2}=\frac{3.5}{d} \frac{(1-\varepsilon)}{\varepsilon^{3}},
\end{aligned}
$$

where $d$ is the mean particle diameter, and $\varepsilon$ is the void fraction [26].

2.3. Grid Generation and Boundary Condition. To solve the governing equations, appropriate grid generation and boundary conditions are specified at all external boundaries based on the following. The 3D model (Figure 3) is meshed into tetrahedral grid (Figure 4), which has about 920538 elements and 164675 nodes for the 3D computational grid. The mesh density is increased appropriately to improve the computational convergent velocity. The technique of finite volume is selected to solve the governing equations. Frequently, suitable values of the underrelaxation factors are adopted to assure the smooth convergence of the numerical solution. In the 


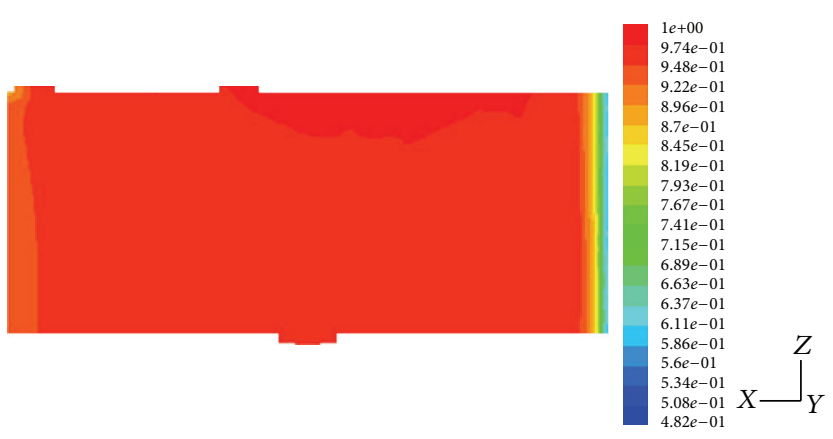

(a) $0.01 \mathrm{MPa}$



(c) $0.002 \mathrm{MPa}$

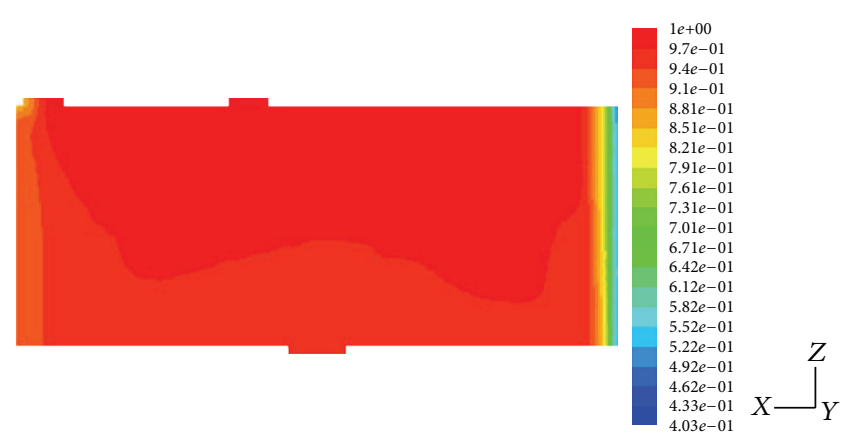

(b) $0.005 \mathrm{MPa}$

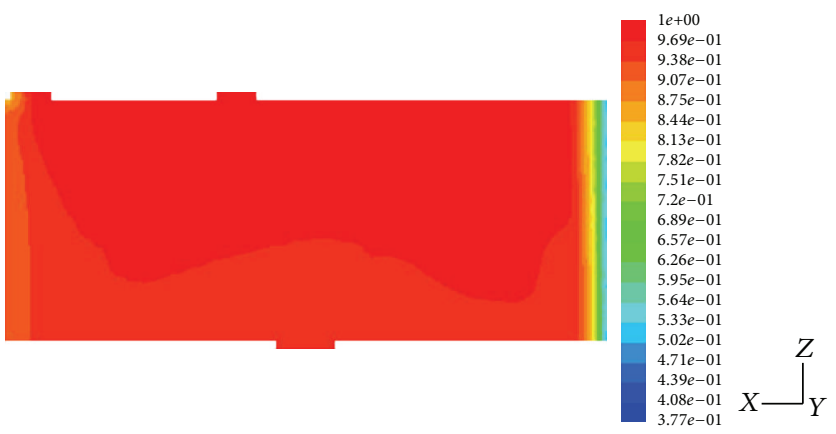

(d) $0.001 \mathrm{MPa}$

FIGURE 5: Contours of the outlet volume fraction of oil in the outlet with different pressures.

TABLE 1: Material properties of the model.

\begin{tabular}{lccc}
\hline Parameter & Unit & Water & Oil \\
\hline Volume fraction & - & $5 \%$ & $95 \%$ \\
Density & $\mathrm{kg} / \mathrm{m}^{3}$ & 1000 & 780 \\
Viscosity & $\mathrm{kg} / \mathrm{ms}$ & 0.001003 & 0.0024 \\
\hline
\end{tabular}

oil-water two-phase flow, the material properties [27] and boundary conditions $[28,29]$ in this research can be seen in Tables 1 and 2.

The material properties and boundary conditions are selected reasonably. The standard $K-\varepsilon$ model is selected, where robustness, economy, and reasonable accuracy for a wide range of turbulent flows explain its popularity in industrial flow and heat transfer simulations.

\section{Results and Discussion}

The multiphase flow in porous media of RPB will be numerical simulated. The effects of operating conditions and configuration on the hydraulic performance of the RPB are investigated to increase the separation efficiency of HIGEE rotating oil purifier.

3.1. Effect of the Inlet Pressure. To understand the feature distribution of inner hydrocyclone separator more clearly, the outlet pressure of hydrocyclone separator is $0.002 \mathrm{MPa}$ which is considered a reference. The static pressure is firstly to be
TABLE 2: Boundary conditions of the model.

\begin{tabular}{lc}
\hline Description & Type \\
\hline Porosity of porous medium & 0.5 \\
Mean particle diameter of porous medium & $0.05 \mathrm{~mm}$ \\
Flow condition of the oil-water mixture & Turbulence \\
Viscous model & $K-\varepsilon$ model \\
Inlet of the oil-water mixture & Pressure inlet \\
Outlet of oil or water & Pressure inlet \\
\hline
\end{tabular}

simulated as shown. This example is identified as follows: the configuration of the RPB is certain. The oil-water mixture inlet is set near to the middle of the lower surface of the RPB, whose diameter is $15 \mathrm{~mm}$. The outlet for water and oil is set in the upper surface, where the oil outlet is in the middle, while the water outlet is in the edge. The diameter of outlets is $10 \mathrm{~mm}$. The rotation speed is $1500 \mathrm{rpm}$. The other conditions are constant. This research investigated the separation efficiency of HIGEE rotating oil purifier by changing the inlet pressure. The sectional drawings are extracted. Contour plot and graph of the outlet volume fraction of oil in the outlet with different pressures are shown in Figures 5 and 6.

Figure 5 displays contour plots of the outlet volume fraction of oil in the outlet with different pressures. As shown in Figure 5(a), the liquid inlet pressure is $0.01 \mathrm{MPa}$. The volume fraction of oil in the oil outlet is not entirely $100 \%$, and the fraction of oil in the water outlet can be about $96 \%$. In Figure 5(b), the liquid inlet pressure is $0.005 \mathrm{MPa}$. 


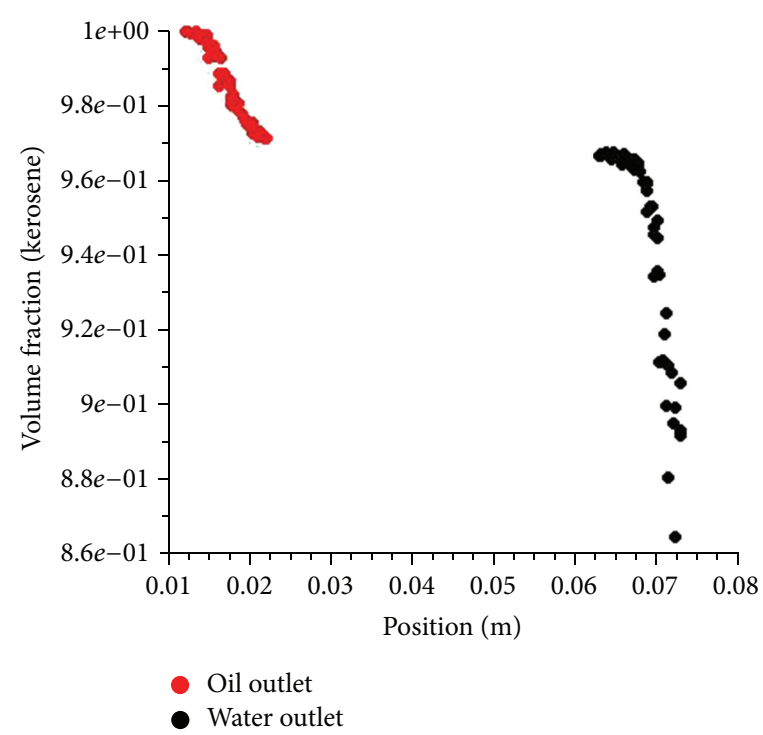

(a) $0.01 \mathrm{MPa}$

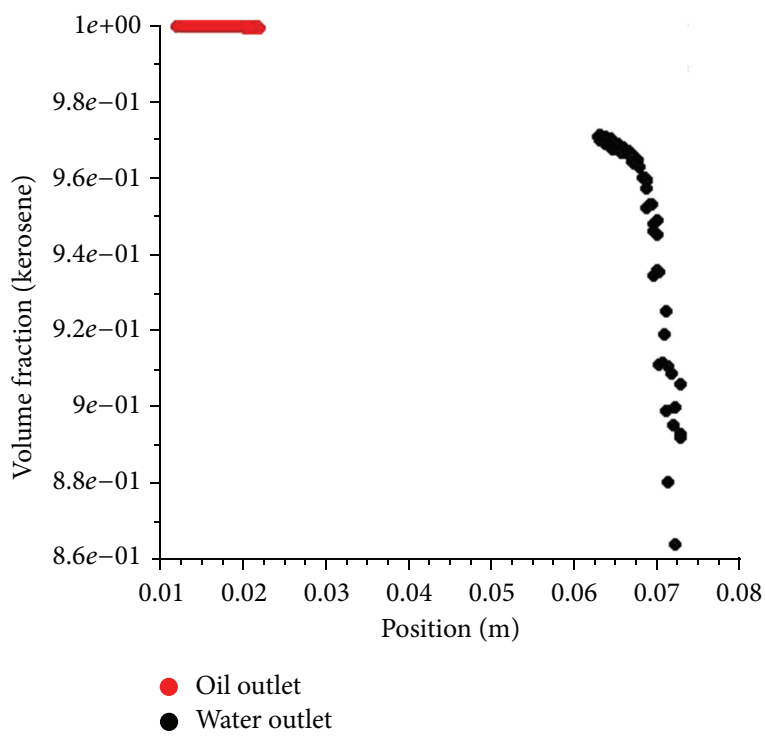

(c) $0.002 \mathrm{MPa}$



(b) $0.005 \mathrm{MPa}$

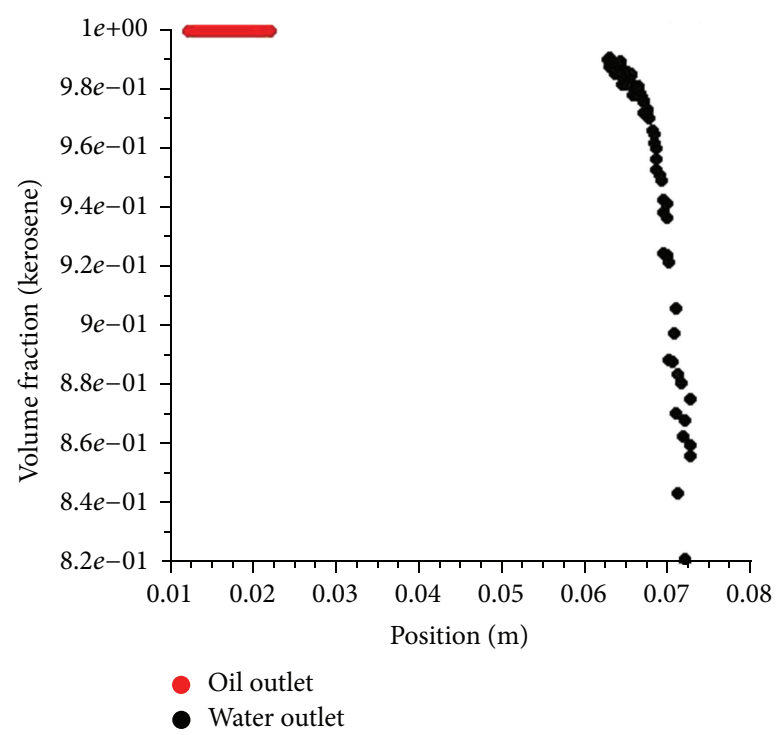

(d) $0.001 \mathrm{MPa}$

FIGURE 6: Graphs of the outlet volume fraction of oil in the outlet with different pressures.

The volume fraction of oil in the oil outlet can be up to $100 \%$, and the fraction of oil in the water outlet can also be about $99 \%$. However, when the liquid inlet pressure is $0.002 \mathrm{MPa}$, the volume fraction of oil in the oil outlet can be up to $100 \%$, as seen in Figure 5(c), and the fraction of oil in the water outlet can also be about $96 \%$. When the liquid inlet pressure is $0.001 \mathrm{MPa}$, the volume fraction of oil in the oil outlet can be up to $100 \%$, which is shown in Figure 5(d), and the fraction of oil in the water outlet can also be about $98 \%$.

Figure 6 displays graphs of the outlet volume fraction of oil in the outlet with different pressures. The volume fraction of oil in the oil outlet can be up to $100 \%$, but it is not entirely $100 \%$, as shown in Figure 6(a), and the fraction of oil in the water outlet can also be about $96 \%$. In Figure 6(b), the volume fraction of oil in the oil outlet can be up to $100 \%$, and it is entirely $100 \%$, but the fraction of oil in the water outlet can also be about more than $99 \%$. When the liquid inlet pressure is $0.002 \mathrm{MPa}$, the volume fraction of oil in the oil outlet can be up to $100 \%$, as shown in Figure 6(c), and it is entirely $100 \%$, but the fraction of oil in the water outlet can also be about less than $96 \%$. As shown in Figure 6(d), the volume fraction of oil in the oil outlet can be up to $100 \%$, and it is entirely $100 \%$, but the fraction of oil in the water outlet can also be about more than $98 \%$.

It can be seen from the simulation results that the inlet pressure has a big effect on the separation efficiency of HIGEE rotating oil purifier. The separation efficiency of HIGEE rotating oil purifier improves with the decreasing of inlet pressure; in particular when the inlet pressure is $0.002 \mathrm{MPa}$, the separation efficiency is the best. The volume fraction of 


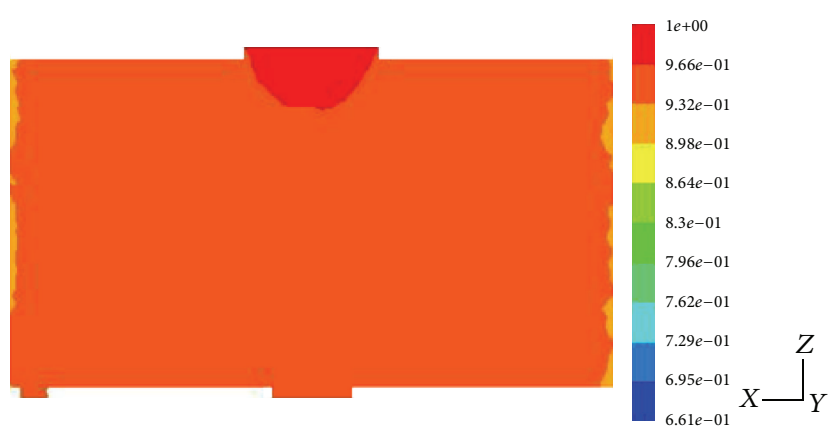

(a) Configuration A
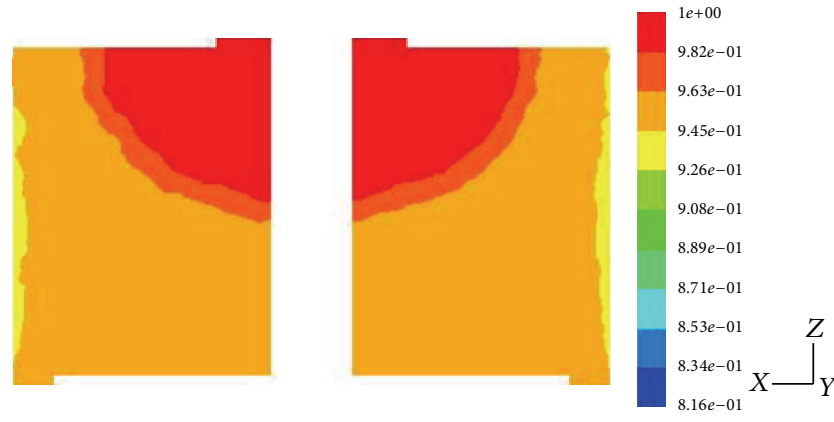

(c) Configuration $\mathrm{C}$

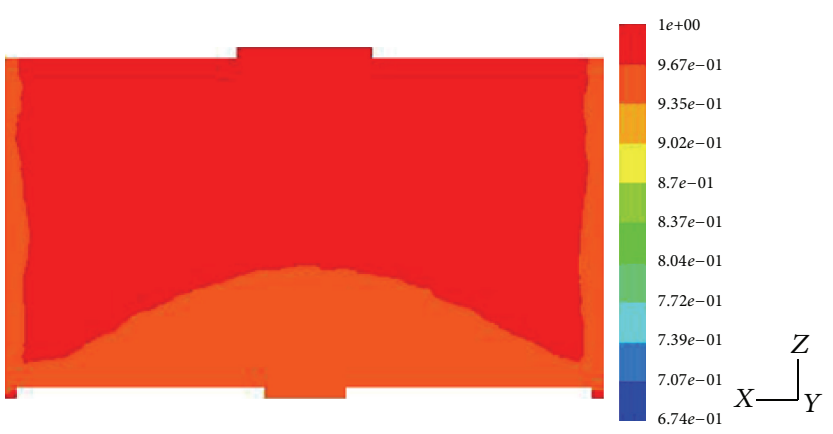

(b) Configuration B
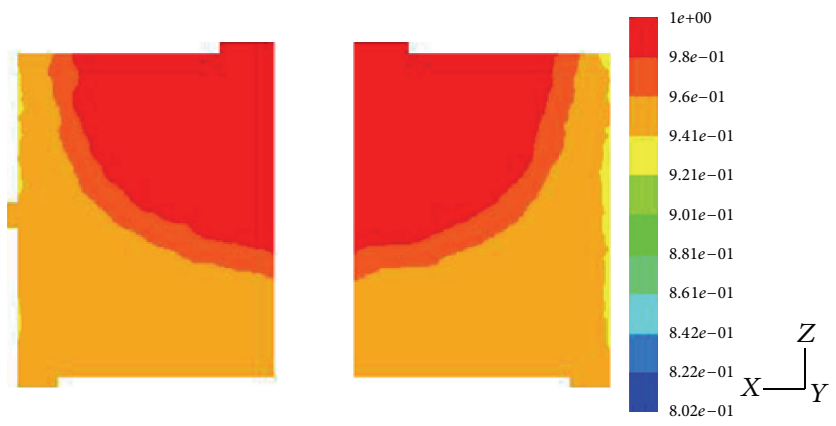

(d) Configuration D

FIGURE 7: Contours of the volume fraction of oil in the outlet with different configurations.

oil in the oil outlet can be up to $100 \%$, and it is entirely $100 \%$, and the fraction of oil in the water outlet can also be about less than $96 \%$, which is better than other inlet pressures.

\subsection{Effect of the Configuration. In order to investigate the} effect of the configuration, the example is identified as follows. The rotation speed is $1500 \mathrm{rpm}$. The inlet pressure is $0.002 \mathrm{MPa}$ with the other boundary conditions unchanged. This research investigated the separation effect of HIGEE rotating oil purifier by changing the configuration, such as the location of the oil-water mixture inlet, oil outlet, and water outlet in the RPB.

Based on previous theoretical and experimental results, fluids of different densities will concentrate on different places accordingly under rotation speed. The fluid of high density concentrates close to the rotation axis, while the fluid of low density concentrates away from the rotation axis. Therefore, four different configurations are investigated in this research. Configuration $A$. The oil-water mixture inlet is placed in the middle of the lower surface of $\mathrm{RPB}$, whose diameter is $15 \mathrm{~mm}$, the water outlet is placed in the outside of the lower surface, whose diameter is $5 \mathrm{~mm}$, and the oil outlet is placed in the middle of the upper surface, whose diameter is $20 \mathrm{~mm}$. Configuration B. The water outlet is also placed in the outside of the lower surface, but its width is $2.5 \mathrm{~mm}$, which is different from Configuration A. Configuration C. The oil-water mixture inlet is placed in the outside of the lower surface, whose width is $7.5 \mathrm{~mm}$, the water outlet is placed in the side of upper, whose diameter is $5 \mathrm{~mm}$, and the oil outlet is placed in the middle of the upper face, whose width is $10 \mathrm{~mm}$. Configuration D. The water outlet is placed in the side of middle, which is different from Configuration C. The sectional drawings are extracted. Contour plot and graph of the outlet volume fraction of oil in the outlet with different configurations are shown in Figures 7 and 8.

Figure 7 displays contour plots of the outlet volume fraction of oil in the outlet with different configurations. As shown in Figure 7(a), the volume fraction of oil in the oil outlet can be up to $100 \%$, and the fraction of oil in the water outlet can also be about 95\%. In Figure 7(b), the volume fraction of oil in the oil outlet can be up to $100 \%$, and the fraction of oil in the water outlet can also be about more than 98\%. Corresponding to the Configuration C, the volume fraction of oil in the oil outlet can be up to $100 \%$, and the fraction of oil in the water outlet can also be about more than 95\% (Figure 7(c)). The volume fraction of oil in the oil outlet can be up to $100 \%$, which is shown in Figure $7(\mathrm{~d})$, and the fraction of oil in the water outlet can also be about less than 95\%.

Figure 8 displays graphs of the outlet volume fraction of oil in the outlet with different configurations. The volume fraction of oil in the oil outlet can be up to $100 \%$, but it is not entirely $100 \%$, which is shown in Figure 8(a). And the fraction of oil in the water outlet can also be about $95 \%$. In Figure $8(\mathrm{~b})$, the volume fraction of oil in the oil outlet can be up to $100 \%$, and it is entirely $100 \%$, but the fraction of oil in the water outlet can also be about more than $98 \%$. Corresponding to Configuration $\mathrm{C}$, the volume fraction of oil in the oil outlet can be up to $100 \%$, and it is entirely $100 \%$, but the fraction of oil in the water outlet can also be about more than $95 \%$ (Figure 8(c)). As shown in Figure 8(d), the volume fraction of oil in the oil outlet can be up to $100 \%$, and it is entirely 




(a) Configuration $\mathrm{A}$



(c) Configuration C

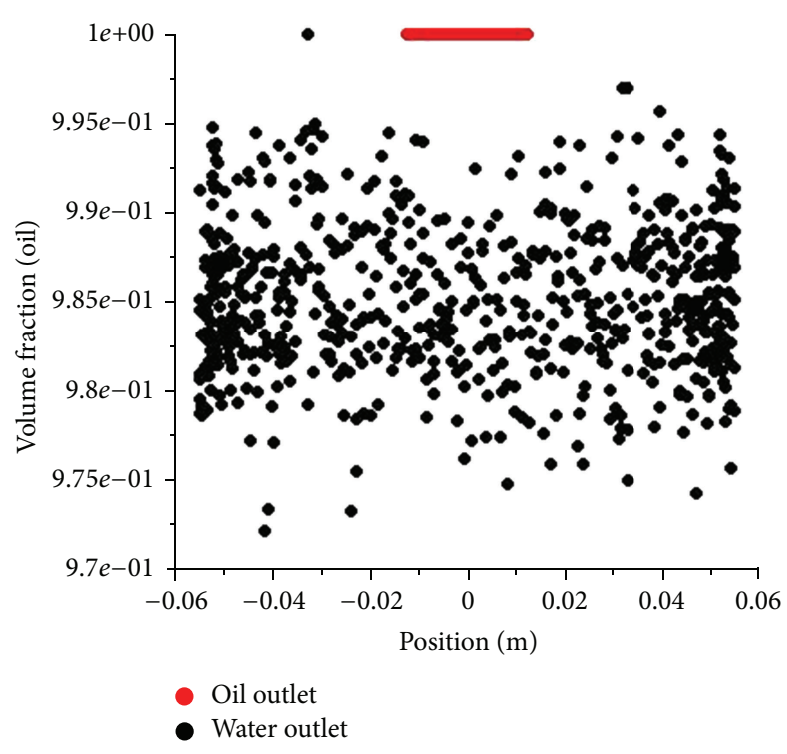

(b) Configuration B

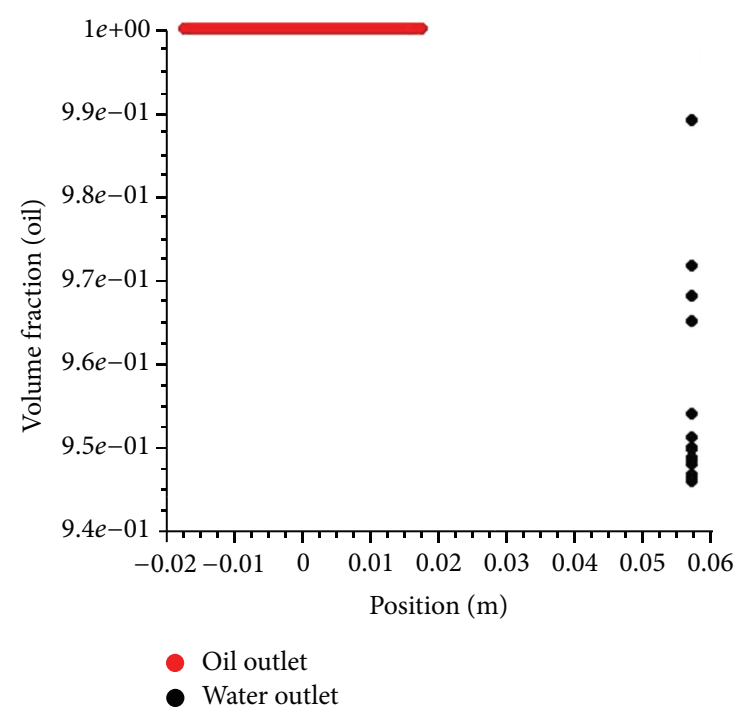

(d) Configuration D

FIGURE 8: Graphs of the volume fraction of oil in the outlet with different configurations.

$100 \%$, but the fraction of oil in the water outlet can also be about less than $95 \%$.

Simulation results show that the simulation results indicate that the separating efficiency of HIGEE rotating oil purifier is greatly affected by the configuration; Configuration $\mathrm{D}$ is the best configuration. In Configuration $\mathrm{D}$, the volume fraction of oil in the oil outlet can be up to $100 \%$, and it is entirely $100 \%$, but the fraction of oil in the water outlet can also be about less than $95 \%$.

3.3. Discussion. Simulation results show that because of different densities, when the oil from preliminary purification goes through the HIGEE field, the oil-which has a low density outflows from the oil outlet in the middle, where it is near the rotation axis, while water which has a high density outflows from the water outlet is in the edge, where it is far away from the oil outlet.

Applying the inlet pressure is for applying an inlet velocity to the oil from preliminary purification. When the inlet pressure is small, which can increase the residence time of the oil-water mixture, therefore, the fluid from preliminary purification can make a good contact with hydrophilic material in the RPB to get better separation efficiency. The separation efficiency of HIGEE rotating oil purifier increases with the decreasing of inlet pressure; in particular when the inlet pressure is $0.002 \mathrm{MPa}$, the separation efficiency is the best. However, a small pressure is not the best choice for the 
inlet pressure. The working hours will last long when the inlet velocity is very small.

The separating efficiency of HIGEE rotating oil purifier is greatly affected by the configurations. Configuration $\mathrm{D}$ is the best configuration. The layout of liquid inlet, oil outlet, and water outlet of the RPB significantly affected the separating efficiency. When the liquid inlet is placed in the outside of the lower surface of RPB; oil outlet is placed in the upper surface, where it is near the rotation axis; and water outlet is placed in the middle of the side of the RPB, where it is far away from the oil outlet, the corresponding separating efficiency was the best. In Configuration D the volume fraction of oil in the oil outlet can be up to $100 \%$, and it is entirely $100 \%$, but the fraction of oil in the water outlet can also be about less than $95 \%$, which is better than other configurations.

\section{Conclusions}

Unlike previous experimental research, numerical simulation is employed in this paper to analyze the flow characteristics inside the RPB, and related conclusions are got.

(1) The oil-water two-phase flow is simulated based on the 3D model of the RPB which is established in Gambit.

(2) The operating conditions on the hydraulic performance of the RPB are investigated. Inlet pressure has big effect on the separation efficiency of HIGEE rotating oil purifier. The separation efficiency of HIGEE rotating oil purifier increases with the decreasing of inlet pressure; in particular when the inlet pressure is $0.002 \mathrm{MPa}$, the separation efficiency is the best.

(3) Simulation results also show that the separating efficiency of HIGEE rotating oil purifier is greatly affected by the configuration, especially the layout of liquid inlet, oil outlet, and water outlet in the RPB. When the liquid inlet is placed in the outside of the lower surface of RPB; oil outlet is placed in the upper surface, where it is near to the rotation axis; and water outlet is placed in the middle of the side of the RPB, which it is far away from the oil outlet, the corresponding separating efficiency is the best.

Compared with theoretical analysis and experimental research, numerical simulation has provided an easy and effective method to design and optimize the HIGEE rotating oil purifier and other mechanical devices, which was widely used in resources and environmental systems. In order to certificate the numerical results, corresponding experiments need to be investigated in the future work.

\section{Acknowledgments}

This research was funded by the Natural Science Foundations of China (no. 51075007), National High-tech R\&D (863) Program (no. 2012AA091103), and The Importation and Development of High-Caliber Talents Project of Beijing Municipal Institutions (CIT\&TCD 20130316).

\section{References}

[1] Z. X. Xia, Contamination Control of Hydraulic System, China Machine Press, Beijing, 1992.

[2] C. Ramshaw, "'HiGee' distillation-An example of process intensification," Chemical Engineering Science, vol. 389, pp. 13-14, 1983.

[3] M. Keyvani and N. C. Gardner, "Operating characteristics of rotating beds," Chemical Engineering Progress, vol. 85, no. 9, pp. 48-52, 1989.

[4] H. S. Liu, C. C. Lin, S. C. Wu, and H. W. Hsu, "Characteristics of a rotating packed bed," Industrial and Engineering Chemistry Research, vol. 35, no. 10, pp. 3590-3596, 1996.

[5] D. P. Rao, A. Bhowal, and P. S. Goswami, "Process intensification in rotating packed beds (HIGee): an Appraisal," Industrial and Engineering Chemistry Research, vol. 43, no. 4, pp. 1150-1162, 2004.

[6] X. Li, Y. Liu, Z. Li, and X. Wang, "Continues distillation experiment with rotating packed bed," Chinese Journal of Chemical Engineering, vol. 16, no. 4, pp. 656-662, 2008.

[7] H. Ji, S. L. Nie, H. M. Sun, and X. H. Tang, "Research on on-line purification of hydraulic oil based on high gravity technology," Chinese Hydraulics \& Pneumatics, no. 11, pp. 1-6, 2011.

[8] C. Zheng, K. Guo, Y. Feng, C. Yang, and N. C. Gardner, "Pressure drop of centripetal gas flow through rotating beds," Industrial and Engineering Chemistry Research, vol. 39, no. 3, pp. 829-834, 2000.

[9] A. Basic and M. P. Dudukovic, "Liquid holdup in rotating packed beds: examination of the film flow assumption," AIChE Journal, vol. 41, no. 2, pp. 301-316, 1995.

[10] M. J. Lockett, "Flooding of rotating structured packing and its application to conventional packed columns," Trans IChemE, vol. 73, pp. 379-384, 1995.

[11] K. Guo, F. Guo, Y. Feng, J. Chen, C. Zheng, and N. C. Gardner, "Synchronous visual and RTD study on liquid flow in rotating packed-bed contractor," Chemical Engineering Science, vol. 55, no. 9, pp. 1699-1706, 2000.

[12] C. Ramshaw and R. H. Mallinson, "Mass transfer process," U.S. Patent 4, vol. 283, no. 255, 1981.

[13] H. H. Tung and R. S. H. Mah, "Modeling liquid mass tranfer in HIGee separtion process," Chemical Engineering Communications, vol. 39, no. 1-6, pp. 147-153, 1985.

[14] S. Munjal, M. P. Dudukovć, and P. Ramachandran, "Masstransfer in rotating packed beds-I. Development of gas-liquid and liquid-solid mass-transfer correlations," Chemical Engineering Science, vol. 44, no. 10, pp. 2245-2256, 1989.

[15] S. Munjal, M. P. Duduković, and P. Ramachandran, "Masstransfer in rotating packed beds-II. Experimental results and comparison with theory and gravity flow," Chemical Engineering Science, vol. 44, no. 10, pp. 2257-2268, 1989.

[16] M. Kumar and N. C. Gardner, "Operating characteristics of rotating beds," Chemical Engineering Science, vol. 85, no. 9, pp. 48-52, 1989.

[17] M. P. Kumar and D. P. Rao, "Studies on a high-gravity gas-liquid contactor," Industrial Engineering Chemistry Research, vol. 29, no. 5, pp. 917-920, 1990.

[18] S. P. Singh, J. H. Wilson, R. M. Counce et al., "Removal of volatile organic compounds from groundwater using a rotary air stripper," Industrial and Engineering Chemistry Research, vol. 31, no. 2, pp. 574-580, 1992. 
[19] Y. H. Chen, C. Y. Chang, W. L. Su et al., "Modeling ozone contacting process in a rotating packed bed," Industrial and Engineering Chemistry Research, vol. 43, no. 1, pp. 228-236, 2004.

[20] Y. S. Chen, C. C. Lin, and H. S. Liu, "Mass tranfer in a rotationg packed bed with viscous newtonian and non-newtonian fluids," Industrial Engineering Chemistry Research, vol. 44, no. 4, pp. 1043-1051, 2005.

[21] Y. S. Chen, C. C. Lin, and H. S. Liu, "Mass tranfer in a rotationg packed bed with viscous radii of the bed," Industrial Engineering Chemistry Research, vol. 44, no. 20, pp. 7868-7875, 2005.

[22] J. R. Burns and C. Ramshaw, "Process intensification: visual study of liquid maldistribution in rotating packed beds," Chemical Engineering Science, vol. 51, no. 8, pp. 1347-1352, 1996.

[23] L. J. Guo, Two-Phase and multiPhase Hydrodynamics, Xian Jiaotong University Press, Xi'an, China, 2002.

[24] X. Y. Kong, Advanced Mechanics of Fluid Flow in Porous Media, Chinese Science and Technology University Press, Hefei, China, 2010.

[25] L. S. Cheng, Advanced Mechanics of Fluid Flow in Porous Media, Petroleum Industry Press, Beijng, China, 2010.

[26] L. Fan, R.T. Hai, W. X. Wang, Z. Lu, and Z. M. Yang, "Application of computational fluid dynamic to model the hydraulic performance of subsurface flow wetlands," Journal of Environmental Sciences, vol. 20, no. 12, pp. 1415-1422, 2008.

[27] Z. Wen, L. C. Shi, and Y. R. Ren, The Flent Computational Dynamics Application Guide, Tsinghua University Press, Beijing, China, 2009.

[28] J. C. Sheng, Hydraulic Fluid Mechanics, Mechanical Industry Press, Beijing, China, 1980.

[29] Y. Yu, J. M. Zhang, and L. T. Jiang, Fluent Introductory and Advanced Course, Beijing Institute of Technology Press, Beijing, China, 2011. 




Advances in

Operations Research

mansans

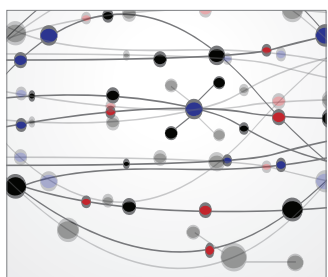

The Scientific World Journal
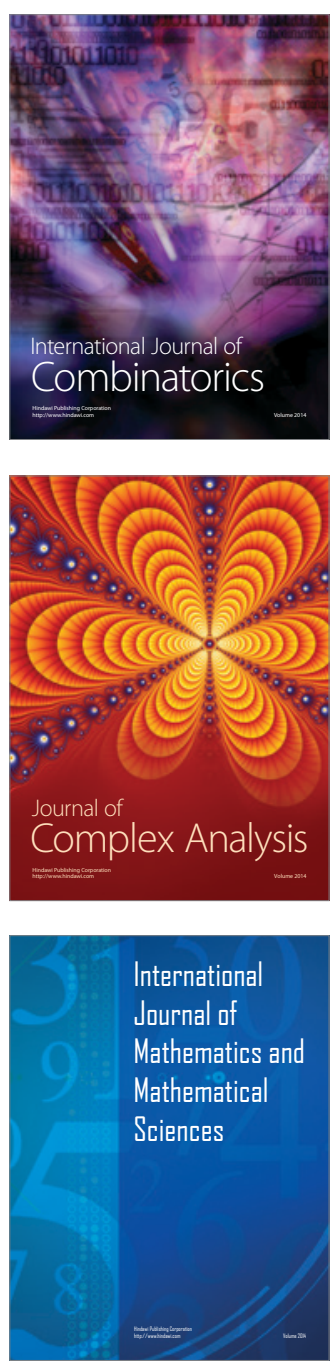
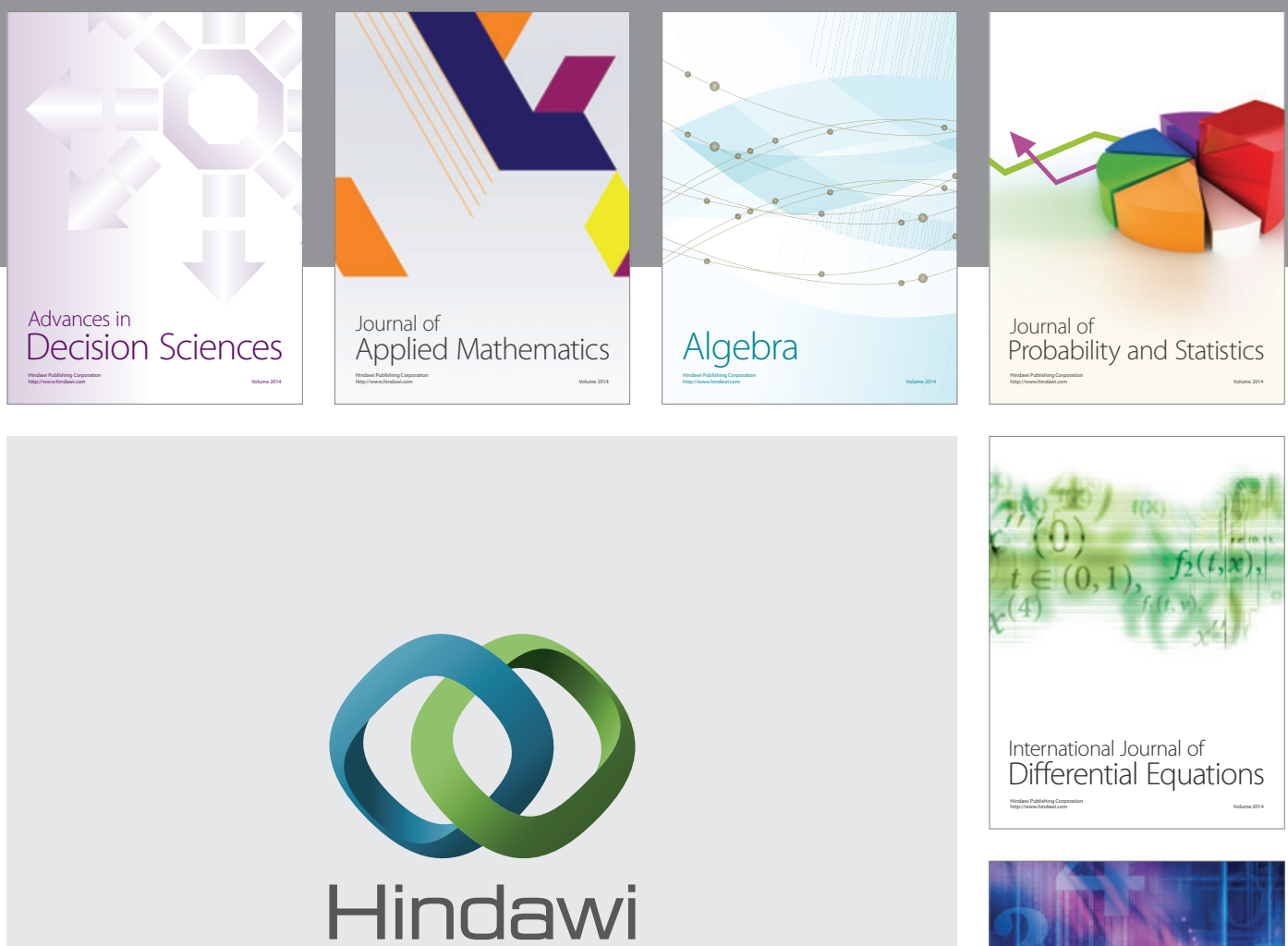

Submit your manuscripts at http://www.hindawi.com
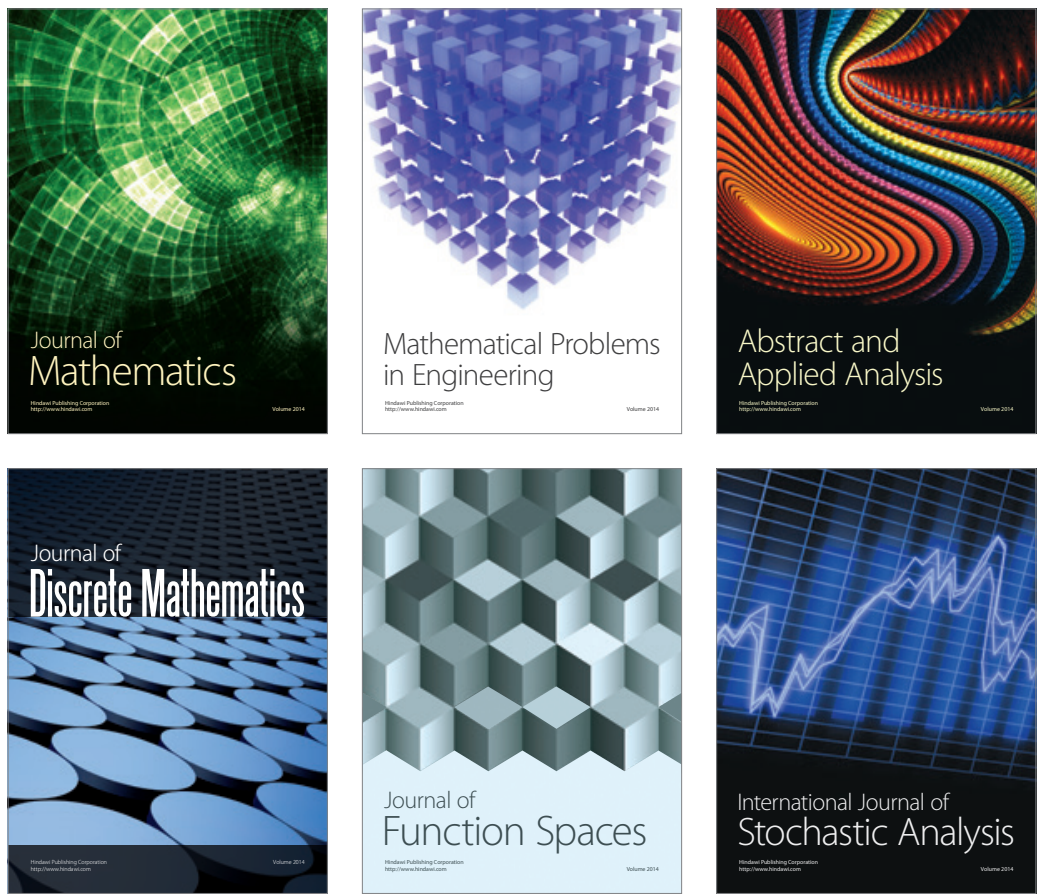

Journal of

Function Spaces




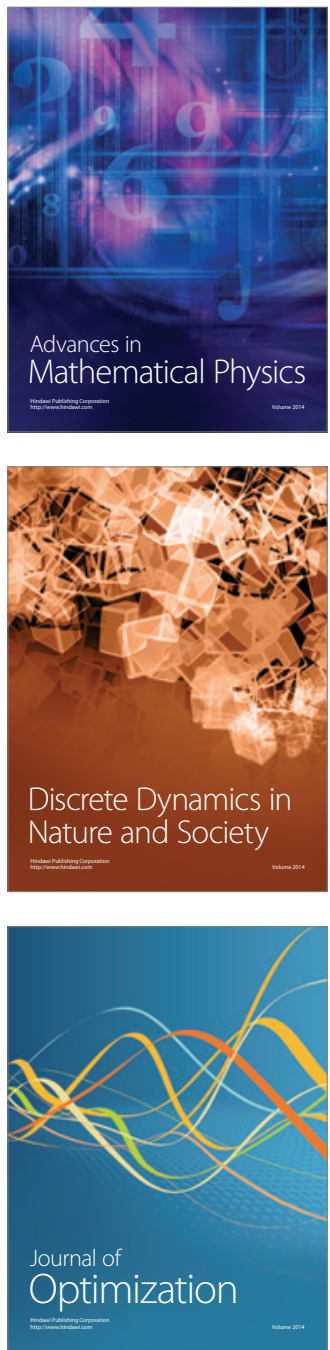